\title{
Prevalence and risk factors of malaria among children in southern highland Rwanda
}

\author{
Jean-Bosco Gahutu ${ }^{1 *}{ }^{*}$, Christian Steininger ${ }^{2+}$, Cyprien Shyirambere ${ }^{1}$, Irene Zeile ${ }^{2}$, Neniling Cwinya-Ay ${ }^{1}$, \\ Ina Danquah ${ }^{2}$, Christoph H Larsen ${ }^{3}$, Teunis A Eggelte ${ }^{4}$, Aline Uwimana ${ }^{5}$, Corine Karema ${ }^{5}$, Andre Musemakweri ${ }^{1}$, \\ Gundel Harms ${ }^{2}$ and Frank P Mockenhaupt ${ }^{2}$
}

\begin{abstract}
Background: Increased control has produced remarkable reductions of malaria in some parts of sub-Saharan Africa, including Rwanda. In the southern highlands, near the district capital of Butare (altitude, 1,768 m), a combined community-and facility-based survey on Plasmodium infection was conducted early in 2010.
\end{abstract}

Methods: A total of 749 children below five years of age were examined including 545 randomly selected from 24 villages, 103 attending the health centre in charge, and 101 at the referral district hospital. Clinical, parasitological, haematological, and socio-economic data were collected.

Results: Plasmodium falciparum infection (mean multiplicity, 2.08) was identified by microscopy and PCR in $11.7 \%$ and $16.7 \%$, respectively; $5.5 \%$ of the children had malaria. PCR-based $P$. falciparum prevalence ranged between 0 and $38.5 \%$ in the villages, and was $21.4 \%$ in the health centre, and $14.9 \%$ in the hospital. Independent predictors of infection included increasing age, low mid-upper arm circumference, absence of several household assets, reported recent intake of artemether-lumefantrine, and chloroquine in plasma, measured by ELISA. Self-reported bed net use (58\%) reduced infection only in univariate analysis. In the communities, most infections were seemingly asymptomatic but anaemia was observed in $82 \%$ and $28 \%$ of children with and without parasitaemia, respectively, the effect increasing with parasite density, and significant also for submicroscopic infections.

Conclusions: Plasmodium falciparum infection in the highlands surrounding Butare, Rwanda, is seen in one out of six children under five years of age. The abundance of seemingly asymptomatic infections in the community forms a reservoir for transmission in this epidemic-prone area. Risk factors suggestive of low socio-economic status and insufficient effectiveness of self-reported bed net use refer to areas of improvable intervention.

\section{Background}

Recent years have seen a substantial increase in malaria control activities. Particularly in East Africa, growing evidence suggests a decline in malaria transmission, morbidity and mortality over the last decade [1-5]. Control measures considered vital to this improvement are the deployment of artemisinin-based combination treatment (ACT), distribution of long-lasting insecticide-treated nets (LLINs), and indoor residual spraying $[3,6]$.

Rwanda is a prime example for the impact malaria control can have. Since 2000, several million insecticide

\footnotetext{
* Correspondence: jgahutu@nur.ac.rw

+ Contributed equally

'Butare University Teaching Hospital, Faculty of Medicine, National University of Rwanda, Butare, Rwanda

Full list of author information is available at the end of the article
}

treated nets (ITNs) have been distributed (mostly LLINs) increasing the percentage of the population ( 10 million) covered by nets to potentially $\geq 70 \%$. In parallel, ACTs have been dispensed on a large scale. In $2007,56 \%$ of households were considered to own a net and $56 \%$ of children to sleep under one [4]. Surveillance and health facility based data indicate that by 2007-2008 these efforts were associated with approximately $50 \%$ or higher declines in confirmed outpatient cases, inpatient cases, and deaths due to malaria in children $<5$ years old $[4,7]$.

While this progress does not appear to be questionable, the extent of the declines as deduced from facility-based data might differ at community level. For instance, community-level case management programmes [8] have been reported to shift primary treatment from health

\section{Biomed Central}


centres to villages and thus decrease the health-facility burden [9]. Such a trend, however, does not necessarily reflect the situation in the community $[10,11]$.

One aim of the present study was therefore to provide up-to-date malariologic data at the levels of community, health centre, and district hospital for a highland area in southern Rwanda from where no published material exists so far. In addition, the study aimed at identifying (modifiable) factors associated with Plasmodium infection and malaria in this population.

\section{Methods}

\section{Study area and sampling}

Butare (population approximately 100,000; altitude $1,768 \mathrm{~m}$ ) is the capital of Huye district, southern province of Rwanda. Located on the central plateau of Rwanda (Figure 1; average altitude, 1,700 m; yearly rainfall, $1,200 \mathrm{~mm}$; mean temperature, $19^{\circ} \mathrm{C}$ ), Butare is surrounded by densely populated farmland hills. Despite two rainy seasons (October-November; MarchMay), the area is prone to drought. The present study was conducted from January 18 to March 26, 2010 but the rainy season started as early as late January in this year.

Rwanda has a mandatory health insurance system in which the mutual health insurance scheme (mutuelle de santé ) is the most widespread. At an annual cost of 1,000 Rwandan Francs (1.28 €; February 2010) per capita, treatment of common diseases is basically free of charge including utilization of district and provincial hospitals provided there is adherence to a strict referral system [12]. Governmental health services in Butare area are provided by several primary health centres, Kabutare district hospital and Butare University Teaching Hospital (CHUB, Centre Hospitalier Universitaire de Butare ).

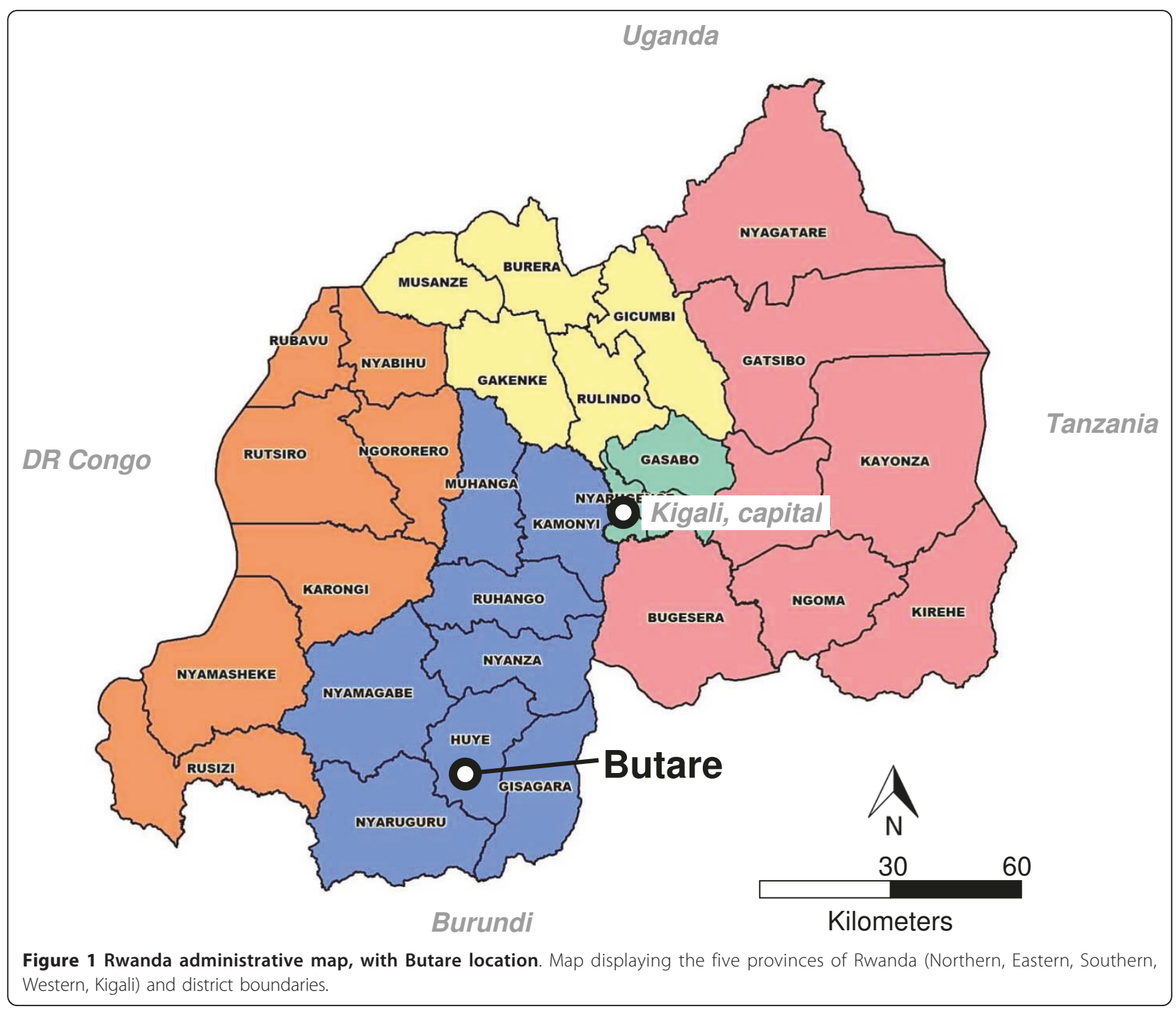


The study was designed as a cross-sectional survey to assess the prevalences of malaria, HIV, and soil-transmitted helminths in children under five years of age in the CHUB catchment area, i.e. at the levels of community, health centre, and district hospital. The present report focuses on the malaria situation. For the community level, the neighbouring rural Huye subdistrict (sector ; population approximately 20,000) was chosen (Figure 2). Based on most recent census data, each 25 households were randomly chosen in a total of 24 randomly selected villages. Community health workers visited these households, randomly selected one child per family, and asked the child to be presented to the study team located at Sovu health centre (or a non-permanently staffed branch) on a scheduled (usually next) day. Thereby, balanced recruitment into the age strata $<1,1$ $<2,2<3,3<4$, and $4<5$ years was aimed at. In parallel, $\geq 100$ paediatric patients aged five years or less and presenting at the primary Sovu health centre and at the referral Kabutare district hospital, i.e. the health facilities serving this population, were successively recruited. All children's parents were thoroughly informed on the purpose and procedures of the study, and recruitment was preceded by HIV pre-counselling and obtaining informed written consent. The study was reviewed and approved by the National Ethics Committee, Republic of Rwanda.

\section{Examinations}

Brief questionnaires were filled in on socio-economic aspects of the children's families including household assets; specification of bed nets, e.g. impregnated or not, was omitted. All children were examined by a physician, a medical history obtained, and a venous blood sample collected. Age, sex, weight, height, mid-upper arm circumference (MUAC), and fever (axillary temperature $\left.\geq 37.5^{\circ} \mathrm{C}\right)$ were documented. Haemoglobin $(\mathrm{Hb})$ levels were measured by a HemoCue photometer (Angelholm, Sweden). Anaemia was defined as an $\mathrm{Hb}$ level $<11 \mathrm{~g} /$ dL. Intestinal parasites were screened for by direct wet

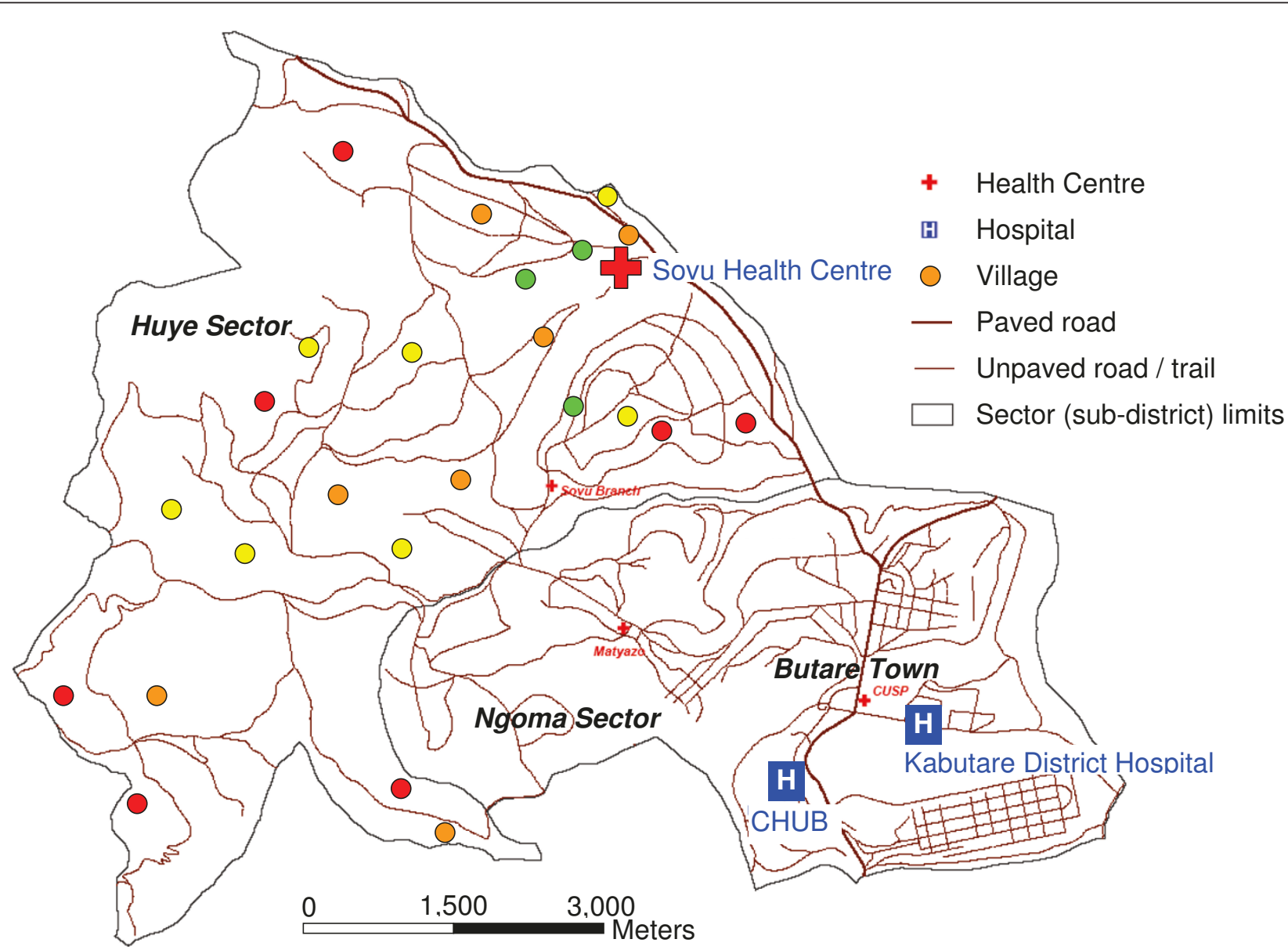

Figure 2 Study area, Butare town and Huye sector. Community children were recruited in 24 villages in the rural Huye sector (subdistrict), and patients at Sovu Health Centre and Kabutare district hospital. The prevalence of $P$. falciparum infection (PCR) among children under five years of age in the villages (sample sizes, 18-26) is marked by colour: green, $<5 \%$; yellow, $5 \leq 10 \%$; orange, $10 \leq 20 \%$; red, $>20 \%$. 
mount microscopical stool examination, and urinary tract infection by dipstick (Multistix 10 SG, Bayer, Germany). Malaria parasites were counted per 200 white blood cells (WBCs) on Giemsa-stained thick blood films, while the patient was waiting. Children with malaria parasites were treated with artemether-lumefantrine. Other diseases were treated according to Rwanda health authority guidelines [13]. Following duplicate readings per $200 \mathrm{WBCs}$ at the $\mathrm{CHUB}$ central laboratory and the Institute of Tropical Medicine \& International Health in Berlin, the definite parasite density was calculated on the basis of a putative mean WBC count of $8,000 / \mu \mathrm{L}$. These data were used for analysis. Malaria was defined as any microscopically visible parasitaemia plus fever or a history of fever within the preceding 48 hours. DNA was extracted (Qiamp blood kit; Qiagen, Germany), and Plasmodium species and submicroscopic infections were identified by semi-nested multiplex polymerase chain reaction (PCR) assays [14]. For all PCR positive samples, sequences corresponding to the allelic families of the Plasmodium falciparum merozoite surface protein 1 ( $m s p 1$ ) block 2 (K1, Mad20, Ro33) and of $m s p 2$ block 3 (FC27, IC) were amplified in five separate PCR assays [15]. Size variation within the alleles can be used to discriminate different parasite clones by PCR fragment length polymorphism, visualised on 3\% GTG $^{\circledR}$-agarose gels (Biozym, Germany) and analysed using GeneSnap software (SynGene, UK). In case of negative or inconclusive PCR results, assays were repeated maximally twice. Multiplicity of infection (MOI) was calculated as the highest number of fragments for either $m s p 1$ or $m s p 2$. Residual (pre-treatment) concentrations of chloroquine and pyrimethamine (indicative of sulphadoxine-pyrimethamine) in plasma were determined by ELISA with limits of detection of $5 \mathrm{ng} /$ $\mathrm{mL}$ and $10 \mathrm{ng} / \mathrm{mL}$, respectively [16].

\section{Statistical analysis}

Data analysis was performed using Statview 5.0 (SAS Institute Inc.). Continuous variables were compared between groups by the non-parametric Mann-Whitney or Kruskal-Wallis tests, and proportions by $\chi^{2}$ test or Fisher's exact test. Odds ratios (ORs) and 95\% confidence intervals (95\% CIs) were computed. Non-parametric ordinal regression analysis was performed to assess significantly differential effects of independent factors, e.g. infection, on a dependent variable, e.g. $\mathrm{Hb}$ levels, between groups. Despite non-parametric comparisons and for tangibility, parasite densities and $\mathrm{Hb}$ concentrations are displayed as geometric mean parasite densities (GMPDs) and means, respectively. Evaluation of determinants of $P$. falciparum infection and malaria was performed by logistic regression analysis. Stepwise backward selection was performed, and final models included those factors that retained statistical significance. A p-value $<0.05$ was considered statistically significant.

\section{Results \\ Study participants}

A total of 749 children were examined including 545 from the rural Huye communities, 103 from Sovu health centre, and 101 from Kabutare district hospital (Table 1). As compared to community children, those attending the health centre or the district hospital were slightly younger. In the communities, fever and a history of fever within the preceding 48 hours were rare $(3 \%$ and $10 \%)$ but common in the health centre $(35 \%, 70 \%)$ and in the district hospital $(27 \%, 48 \%)$. The primary clinical diagnoses differed between the groups (overall, $P<$ 0.0001). In community children, the leading one was gastro-intestinal affection including gastroenteritis, amoebiasis and helminthiasis. In health centre and hospital, the leading primary diagnosis was respiratory tract infection (Table 1). Chloroquine in plasma was found in $3.7 \%(28 / 747)$ of all children at a median concentration of $15 \mathrm{ng} / \mathrm{mL}$ (range, 8-240). Only one child exhibited pyrimethamine in plasma $(50 \mathrm{ng} / \mathrm{mL})$.

The three groups showed large differences in the socio-economic characteristics of the children's families (Table 2). In the communities, most children lived in rural areas, the average monthly family income was low; one third of the parents had no education at all, and almost all worked as farmers or labourers. Accordingly, asset ownership was generally limited. Less than half of the children were covered by any health insurance; for slightly more than half, a bed net was reported to have been used in the preceding night. Compared to that, socio-economic parameters almost consistently indicated better conditions among children attending the health facilities (Table 2). In particular, among health facility attendees, $85 \%$ were covered by a health insurance, and the rate of self-reported bed net use was $71 \%$. Many socio-economic parameters were inter-related. Monthly family income, for instance, was higher in those with a health insurance than in those without (medians, 10,000 vs. 5,000 Rwandan Francs, $P<0.0001$ ) and higher in those using bed nets as compared to non-users $(8,000$ vs. 5,000 Rwandan Francs, $P=0.0003$ ).

\section{Parasitological parameters}

Overall, $16.7 \%$ of all 749 children were found by PCR to harbour P. falciparum , $11.7 \%$ had microscopically visible parasitaemia, and 5.5\% malaria. All microscopically positive samples were also positive by PCR (including one Plasmodium malariae and two Plasmodium ovale mono-infections). The prevalences of $P$. falciparum infections detected by PCR (range, 16-21\%) and of 
Table 1 Characteristics of 749 children from southern highland Rwanda

\begin{tabular}{|c|c|c|c|c|}
\hline Parameter & Huye communities & Sovu Health Centre & Kabutare District Hospital & $P$ \\
\hline No. (\%) & $545(72.8)$ & $103(13.8)$ & $101(13.5)$ & \\
\hline Age (months) & $31.1(1-60)$ & $28.3(1-59)$ & $27.2(1-60)^{*}$ & 0.03 \\
\hline Proportion girls (\%) & 45.5 & 50.5 & 50.5 & 0.48 \\
\hline Weight (kg) & $11.3(3.5-18.8)$ & $10.9(3.8-20.0)$ & $11.0(3.3-19.0)$ & 0.24 \\
\hline Height $(\mathrm{cm})^{\mathrm{a}}$ & $80.0(41-108)$ & $76.1(42-112)^{*}$ & $78.5(52-110)$ & 0.02 \\
\hline $\operatorname{MUAC}(\mathrm{cm})^{\mathrm{b}}$ & $13.7(5.0-18.0)$ & $14.2(10.5-19.0)^{*}$ & $13.6(8.0-18.5) \dagger$ & 0.04 \\
\hline Axillary temperature $\left({ }^{\circ} \mathrm{C}\right)^{c}$ & $36.7(36.0-40.6)$ & $37.4(36.0-40.1)^{*}$ & $37.0(35.8-39.4) \dagger$ & $<0.0001$ \\
\hline Fever (\%) & $3.3(18 / 543)$ & $35.0^{*}$ & $26.7^{*}$ & $<0.0001$ \\
\hline History of fever, last 2 days (\%) & $9.8(49 / 502)$ & $69.4(68 / 98)^{*}$ & $47.5(47 / 99)^{*} \dagger$ & $<0.0001$ \\
\hline $\mathrm{Hb}(\mathrm{g} / \mathrm{dL})^{\mathrm{b}}$ & $11.3(1.7-15.3)$ & $11.4(1.4-16.6)$ & $11.1(4.3-16.8)$ & 0.89 \\
\hline Anaemia $(\mathrm{Hb}<11 \mathrm{~g} / \mathrm{dL}), \%$ & 34.1 & 35.0 & $32.0(32 / 100)$ & 0.89 \\
\hline Severe Anaemia $(H b<7 \mathrm{~g} / \mathrm{dl}), \%$ & 1.8 & 1.9 & $7.0(7 / 100)^{*}$ & 0.01 \\
\hline \multicolumn{5}{|l|}{ Primary diagnosis on examination ${ }^{d}$} \\
\hline Healthy child & 25.4 & $4.3^{*}$ & $1.5^{*}$ & $<0.0001$ \\
\hline Gastro-intestinal tract affection ${ }^{e}$ & 31.9 & 25.0 & $19.4^{*}$ & 0.04 \\
\hline Respiratory tract infection & 7.3 & $34.3^{*}$ & $26.1^{*}$ & $<0.0001$ \\
\hline Malaria (suspected) ${ }^{f}$ & 9.7 & $19.3^{*}$ & 11.9 & 0.0001 \\
\hline Severe malnutrition (clinically) & 8.2 & $1.4^{*}$ & 3.7 & 0.01 \\
\hline Skin infection & 4.6 & 5.7 & 4.5 & 0.67 \\
\hline Burns, wounds, accidents, etc. & 2.1 & 2.1 & $14.9^{*} \dagger$ & $<0.0001$ \\
\hline Severe anaemia (clinically) & 2.4 & 0.7 & 3.0 & 0.41 \\
\hline Conjunctivitis & 1.4 & 1.4 & 3.7 & 0.11 \\
\hline Disability & 1.1 & 0 & $5.2^{*}+$ & $<0.0001$ \\
\hline Oral problems & 0.9 & 1.4 & $3.7^{*}$ & 0.02 \\
\hline Urinary tract infection & 0.6 & $2.9^{*}$ & 2.2 & 0.02 \\
\hline Others, missing data & 4.4 & 1.4 & $0^{*}$ & 0.03 \\
\hline
\end{tabular}

Numerical data are means (range) unless otherwise indicated, and compared by the non-parametric Kruskal Wallis or Mann Whitney $U$ tests. Proportions were compared by $\chi^{2}$ test or Fisher's exact test. MUAC, mid upper arm circumference; Hb, haemoglobin; GMPD, geometric mean parasite density; Malaria, definition a $n=746 ;{ }^{b}, n=748 ;{ }^{c}, n=747 ;{ }^{d}$, as judged by study physician, several diagnoses per child possible; No. of diagnoses: community, $n=658 ;$ health centre, $n=$ 134; hospital, $n=140 .{ }^{e}$, includes gastroenteritis, amoebiasis, helminthiasis, and others; ${ }^{f}$, based on field-based microscopy and clinical judgement. ${ }^{*}$, difference to Huye communities, $P<0.05 ; \dagger$, difference to Sovu health centre, $P<0.05$

microscopically visible parasitaemia (range, 10\%-17\%) did not differ between the groups (Table 3). However, whereas only one quarter of community children with parasitaemia was classified as having malaria, this was the case in all children at the health centre and in most at the district hospital. Likewise, GMPDs were lower in community children as compared to health centre $(P=$ $0.02)$ or, non-significantly, to district hospital $(P=0.40$; Table 3). As for the non-falciparum parasites, $P$. malariae was rare in the community but reached $3 \%$ in the health centre $(P=0.01)$.

In the villages, the prevalence of $P$. falciparum infection (PCR) ranged from 0 to $38.5 \%(P=0.0002$; Figure 2 ). The number of children in these communities allowed age-stratified analysis of parasitological parameters (numbers in age-groups: $<1$ year, $59 ; 1<2$ years, 136; $2<3$ years, $136 ; 3<4$ years, $120 ; 4<5$ years, 94 ). By $\chi^{2}$ test for trend, the prevalences of $P$. falciparum -infection by PCR $(P=0.009)$, of microscopically visible parasitaemia $(P=0.03)$, and of malaria $(P=0.02)$ increased with age (Figure 3). Likewise, the proportion of asymptomatically infected children (PCR positive but no current or history of fever) among all infected children tended to decline with every year of age $(100 \%(6 / 6)$, $88.8 \%$ (16/18), $85.0 \%$ (17/20), 81.0\% (17/21), and 73.9\% (17/23), $P=0.08)$. GMPDs (95\% CIs) did not show a clear trend: in the above age groups, they were 308 (143-663), 1,374 (513-3,680), 1,162 (339-3,975), 3,491 (714-17,063), and 2,061 (781-5,437) parasites/ $\mu \mathrm{L}$, respectively $(P=0.29)$.

Multiplicity of infection (MOI) was successfully typed for $88.8 \%(111 / 125)$ of all $P$. falciparum isolates. MOI ranged from one to five (mean, 2.02; median, 2.0 ); $65 \%$ $(72 / 111)$ of infections were polyclonal $(\mathrm{MOI}>1)$. MOI did not differ between community children and those attending health facilities (Table 3), did not correlate with age (months; Spearman's $r=0.14 ; P=0.33$; community children only, $r=0.19, P=0.20$ ) but was increased in microscopically visible parasitaemia as compared to submicroscopic infections (means, 2.15 vs. 1.67; 
Table 2 Selected socio-economic characteristics in 749 children from southern highland Rwanda

\begin{tabular}{|c|c|c|c|c|}
\hline Parameter & Huye communities & Sovu Health Centre & Kabutare District Hospital & $P$ \\
\hline$\overline{\text { No. }}$ & 545 & 103 & 101 & \\
\hline Rural residence (\%) & $95.1(507 / 533)$ & $73.7(70 / 95)^{*}$ & $66.0(66 / 100) *$ & $<0.0001$ \\
\hline Monthly family income $(\mathrm{RwF})^{\mathrm{a}}$ & $\begin{array}{l}9124 \\
(0-100,000)\end{array}$ & $\begin{array}{l}31,505 \\
(0-300,000) *\end{array}$ & $\begin{array}{l}28,916 \\
(500-350,000) *\end{array}$ & $<0.0001$ \\
\hline \multicolumn{5}{|l|}{ Mothers education (\%) } \\
\hline None & $30.4(165 / 543)$ & $20.4(21 / 103)$ & $14.9(15 / 101)$ & \\
\hline Primary & $67.0(364 / 543)$ & $68.0(70 / 103)$ & $69.3(70 / 101)$ & \\
\hline Secondary or higher & $2.6(14 / 543)$ & $11.7(12 / 103) *$ & $15.8(16 / 101) *$ & $<0.0001$ \\
\hline Mother's occupation farmer/labourer (\%) & $98.7(533 / 540)$ & $92.2(95 / 103) *$ & $78.2(79 / 101) *+$ & $<0.0001$ \\
\hline \multicolumn{5}{|l|}{ Father's education (\%) } \\
\hline None & $36.5(195 / 534)$ & $19.6(20 / 102)$ & $19.8(20 / 101)$ & \\
\hline Primary & $60.1(321 / 534)$ & $67.6(69 / 102)$ & $60.4(61 / 101)$ & \\
\hline Secondary/tertiary & $3.4(18 / 534)$ & $12.7(13 / 102) *$ & $19.8(20 / 101) *$ & $<0.0001$ \\
\hline \multicolumn{5}{|l|}{ Father's occupation (\%) } \\
\hline Farmer/labourer & $86.9(472 / 543)$ & $73.3(74 / 101)$ & $65.0(65 / 100)$ & \\
\hline Else & $5.5(30 / 543)$ & $25.7(26 / 101)$ & $35.0(35 / 100)$ & \\
\hline Died/left/prisoner & $7.6(41 / 543)$ & $1.0(1 / 101) *$ & $0 *$ & $<0.0001$ \\
\hline No. of people/household ${ }^{a}$ & $5.5(2-12)$ & $5.2(3-12)$ & $5.0(2-12) *$ & 0.03 \\
\hline No. of siblings $s^{a}$ & $2.0(0-9)$ & $1.8(0-7)$ & $1.4(0-6) *$ & 0.002 \\
\hline \multicolumn{5}{|l|}{ Household asset present (\%) } \\
\hline Electricity & $1.3(7 / 542)$ & $11.7(12 / 103) *$ & $23.8(24 / 101) *+$ & $<0.0001$ \\
\hline Piped water & $14.3(77 / 540)$ & $6.8(7 / 103) *$ & $37.6(38 / 101) *+$ & $<0.0001$ \\
\hline Radio & $43.2(233 / 539)$ & $67.0(69 / 103) *$ & $77.2(78 / 101) *$ & $<0.0001$ \\
\hline TV & $0.7(4 / 541)$ & $5.9(6 / 102) *$ & $12.9(13 / 101) *$ & $<0.0001$ \\
\hline Cupboard & $8.9(48 / 540)$ & $24.3(25 / 103) *$ & $37.6(38 / 101) *+$ & $<0.0001$ \\
\hline Bicycle & $9.1(49 / 540)$ & $37.9(39 / 103) *$ & $27.7(28 / 101) *$ & $<0.0001$ \\
\hline Motor-bike & $0.6(3 / 540)$ & $2.9(3 / 102)$ & $1.0(1 / 101)$ & 0.07 \\
\hline Fridge & $0(0 / 541)$ & $1.0(1 / 102) *$ & $2.0(2 / 101) *$ & 0.01 \\
\hline Cattle & $13.0(70 / 539)$ & $43.7(45 / 103) *$ & $20.8(21 / 102) *+$ & $<0.0001$ \\
\hline Health insurance present $(\%)^{c}$ & $43.0(234 / 544)$ & $90.2(92 / 102) *$ & $79.3(80 / 101) *+$ & $<0.0001$ \\
\hline Child received any drug in last 2 weeks & $8.3(45 / 545)$ & $13.7(14 / 102)$ & $38.0(38 / 100) *+$ & $<0.0001$ \\
\hline Child used bed net last night (\%) & $52.7(286 / 543)$ & $69.6(71 / 102) *$ & $73.3(74 / 102) *$ & $<0.0001$ \\
\hline Chloroquine in plasma (\%) & $3.7(20 / 545)$ & $4.0(4 / 101)$ & $4.0(4 / 101)$ & 0.98 \\
\hline
\end{tabular}

Numerical data are means (range) unless otherwise indicated, and compared by the non-parametric Kruskal Wallis and Mann Whitney $\mathrm{U}$ tests. Proportions were compared by $\chi^{2}$ test or Fisher's exact test. ${ }^{a}, n=748 ;{ }^{*}$, difference to Huye communities, $P<0.05$; $\dagger$, difference to Sovu health centre, $P<0.05$

Table 3 Parasitological parameters in 749 children from southern highland Rwanda

\begin{tabular}{|c|c|c|c|c|}
\hline Parameter & Huye communities & Sovu Health Centre & Kabutare District Hospital & $P$ \\
\hline No. & 545 & 103 & 101 & \\
\hline Parasitaemia (\%) & 11.2 & 16.5 & 9.9 & 0.25 \\
\hline GMPD (parasites/ $\mu \mathrm{L} ;$ 95\%Cl) & $1574(913-2714)$ & $7603(2127-27185) *$ & $5508(701-43251)$ & 0.04 \\
\hline MOI (mean, range) & $2.05(1-5)$ & $1.95(1-4)$ & $1.92(1-4)$ & 0.92 \\
\hline Malaria (\%) & 2.9 & $16.5 *$ & $7.9 *$ & $<0.0001$ \\
\hline P. falciparum infection, PCR (\%) & 16.1 & 21.4 & 14.9 & 0.37 \\
\hline P. ovale infection, PCR (\%) & 0.9 & 1.9 & 3.0 & 0.22 \\
\hline P. malariae infection, PCR (\%) & 0.2 & $2.9^{*}$ & 2.0 & 0.006 \\
\hline Proportion of submicroscopic infections $(\%, \mathrm{n} / \mathrm{n})$ & $33.7(31 / 92)$ & $22.7(5 / 22)$ & $41.2(7 / 17)$ & 0.45 \\
\hline Child received artemether-lumefantrine in last 2 weeks & 3.1 & 1.0 & 5.9 & 0.13 \\
\hline
\end{tabular}

GMPD, geometric mean parasite density, and MOI, multiplicity of infection, are compared by the non-parametric Kruskal Wallis and Mann Whitney $U$ tests. Proportions were compared by $\chi^{2}$ test or Fisher's exact test. ${ }^{*}$, difference to Huye communities, $P<0.05$; 


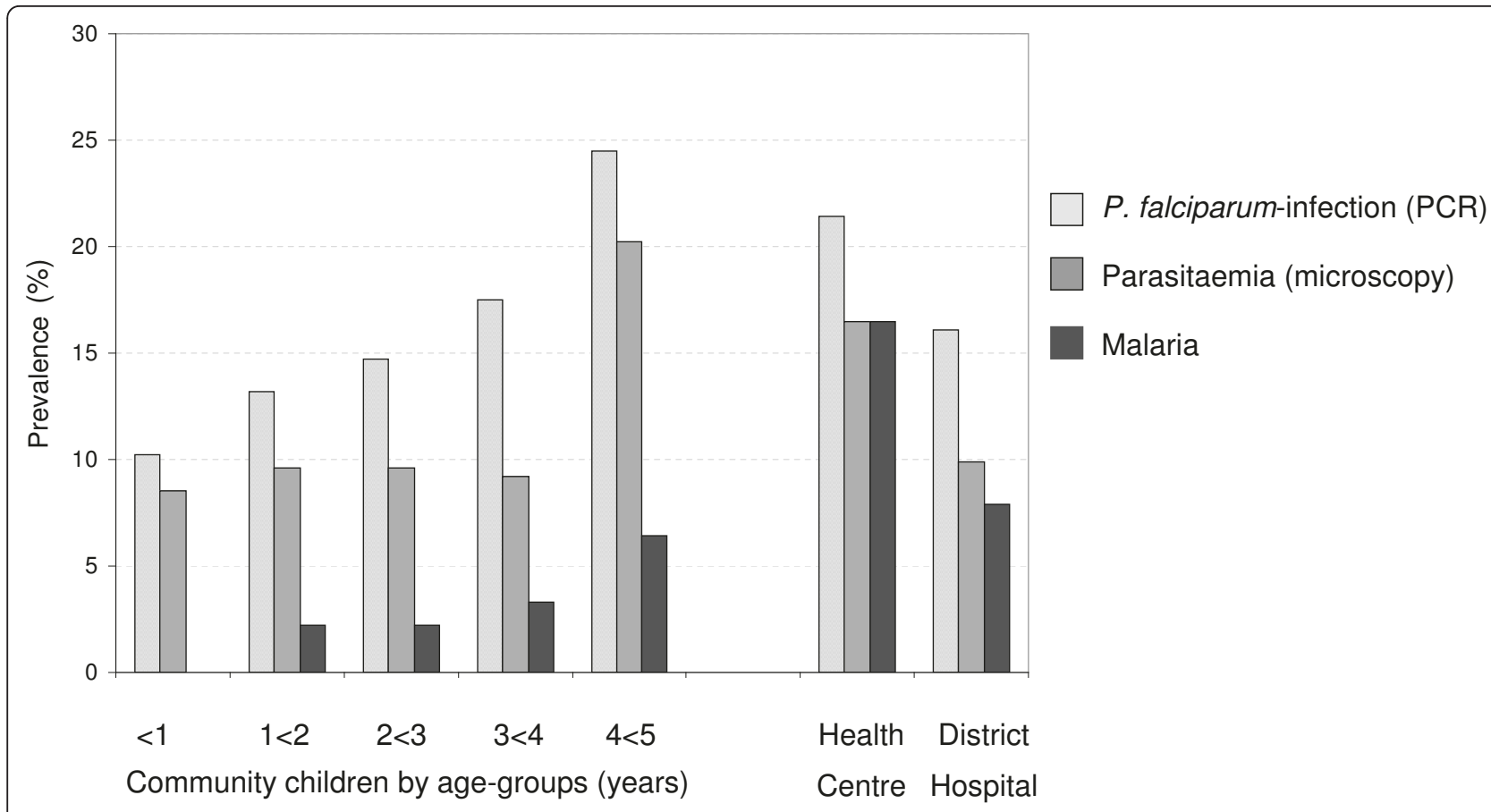

Figure 3 Prevalences of $P$. falciparum infection (PCR), parasitaemia (microscopy) and malaria in 749 children from southern highland Rwanda.

$P=0.03)$, correlated positively with parasite density $(r=$ $0.28, P=0.006)$, and tended to be reduced in former artemether-lumefantrine (AL) recipients as compared to children without such reported intake (means, $1.54 \mathrm{vs.}$ 2.08; $P=0.06$ ).

\section{Factors associated with $P$. falciparum infection and malaria}

Beyond age, a number of factors influenced the presence of infection or malaria. In explorative univariate analysis, socio-economic parameters (Table 2), MUAC, self-reported bed net use, previous AL treatment, and chloroquine in plasma were tested for association with $P$. falciparum infection. The odds of $P$. falciparum was found to increase with increasing age, decreasing MUAC, low educational level, absent father, absence of several household assets, a low family income, lacking use of a bed net, intake of AL within the preceding two weeks (median 7 days before; range, 1-14), and the presence of chloroquine in plasma. For multivariate analysis, univariately associated factors, adjusted for study sub-groups, were entered into a logistic regression model, and subjected to stepwise backward removal (Table 4). Independent predictors of P. falciparum infection included increasing age, health centre attendance, low MUAC, absence of cupboard, radio and bicycle, recent AL intake, and presence of chloroquine in plasma.
For malaria, the same analysis produced the following independently associated factors (aOR (95\% CI)): age (months, 1.02 (1.0-1.05), $P=0.04)$, absent father $(6.53$ (2.15-19.83), $P=0.0009)$, presence of radio $(0.30$ (0.13$0.65), P=0.003)$, recent intake of AL (4.40 (1.24-15.57), $P=0.02)$, and chloroquine in plasma (4.87 (1.43-16.57), $P=0.01)$, adjusted for attendance at health centre (15.93 (6.47-39.26), $P<0.0001)$ or district hospital (9.16 (3.16-26.52), $P<0.0001)$. The role of village among community children lost significance in multivariate analysis (all, $P>0.05$ ). In the above final models, selfreported bed net use showed no association with $P$. falciparum infection (aOR, $0.88(0.56-1.38), P=0.58)$ or malaria (aOR, 0.99 (0.47-2.10), $P=0.98)$.

Limiting multivariate analysis to community children produced basically the same results. However, household possessions of cupboard or bicycle lost significant association with $P$. falciparum infection. In multivariate analysis of current malaria in community children, chloroquine in plasma (prevalence, 3.7\%) lost significant association, and that of age (months) became borderline significant (aOR, 1.03; 95\%CI, 1.0-1.07; $P=0.07$ ).

\section{Clinical manifestations}

Because ordinal regression analyses revealed that $P$. falciparum infection (PCR) in the three groups had significantly differing effects on $\mathrm{Hb}(r=0.6$; standard error $(\mathrm{SE})=0.25 ; P=0.02)$ and body temperature $(r=0.83$; 
Table 4 Univariate and multivariate analysis of factors associated with $P$. falciparum infection (PCR)

\begin{tabular}{|c|c|c|c|c|c|c|c|c|}
\hline \multirow[t]{2}{*}{ Parameter } & \multirow[t]{2}{*}{ No. } & \multirow[t]{2}{*}{ Proportion infected (\%) } & \multicolumn{3}{|c|}{ Univariate analysis } & \multicolumn{3}{|c|}{ Multivariate analysis } \\
\hline & & & OR & $95 \% \mathrm{Cl}$ & $P$ & aOR & $95 \% \mathrm{Cl}$ & $P$ \\
\hline \multicolumn{9}{|l|}{ Group } \\
\hline Huye communities & 545 & 16.1 & 1 & & & 1 & & \\
\hline Health Centre & 101 & 21.4 & 1.41 & $0.81-2.45$ & 0.20 & 2.74 & $1.44-5.19$ & 0.002 \\
\hline District Hospital & 103 & 14.9 & 0.91 & $0.48-1.70$ & 0.74 & 1.72 & $0.86-3.44$ & 0.12 \\
\hline Age (months) & 749 & n.a. & 1.02 & $1.01-1.03$ & 0.001 & 1.03 & $1.01-1.04$ & $<0.0001$ \\
\hline MUAC (cm) & 748 & n.a. & 0.88 & $0.79-0.99$ & 0.03 & 0.83 & 072-0.95 & 0.008 \\
\hline \multicolumn{9}{|l|}{ Mother's education } \\
\hline None & 201 & 20.9 & 1 & & & & & \\
\hline Primary & 504 & 16.1 & 0.72 & $0.47-1.12$ & 0.13 & & & \\
\hline Secondary/tertiary & 42 & 4.8 & 0.19 & $0.02-0.79$ & 0.01 & & & \\
\hline \multicolumn{9}{|l|}{ Father's education } \\
\hline None & 235 & 23.0 & 1 & & & & & \\
\hline Primary & 451 & 14.2 & 0.55 & $0.36-0.85$ & 0.004 & & & \\
\hline Secondary/tertiary & 51 & 7.8 & 0.29 & $0.07-0.84$ & 0.01 & & & \\
\hline \multicolumn{9}{|l|}{ Father's occupation } \\
\hline Farmer/labourer & 611 & 16.9 & 1 & & & & & \\
\hline Else & 91 & 8.8 & 0.48 & $0.21-1.05$ & 0.05 & & & \\
\hline Died/left/prisoner & 42 & 31.0 & 2.21 & $1.05-4.60$ & 0.02 & & & \\
\hline \multicolumn{9}{|l|}{ Pipe-born water } \\
\hline No & 622 & 18.3 & 1 & & & & & \\
\hline Yes & 122 & 7.4 & 0.35 & $0.16-0.75$ & 0.003 & & & \\
\hline \multicolumn{9}{|l|}{ Cupboard } \\
\hline No & 633 & 18.3 & 1 & & & 1 & & \\
\hline Yes & 111 & 6.3 & 0.30 & $0.11-0.66$ & 0.002 & 0.37 & $0.15-0.92$ & 0.03 \\
\hline \multicolumn{9}{|l|}{ Radio } \\
\hline No & 363 & 23.7 & 1 & & & 1 & & \\
\hline Yes & 380 & 9.5 & 0.34 & $0.22-0.52$ & $<0.0001$ & 0.40 & $0.24-0.66$ & 0.0003 \\
\hline \multicolumn{9}{|l|}{ Bicycle } \\
\hline No & 628 & 18.5 & 1 & & & 1 & & \\
\hline Yes & 116 & 6.0 & 0.28 & $0.11-0.63$ & 0.0009 & 0.38 & $0.15-1.0$ & 0.049 \\
\hline \multicolumn{9}{|l|}{ Household income } \\
\hline$>=5000$ RwF (median) & 498 & 14.5 & 1 & & & & & \\
\hline$<5000$ RwF & 250 & 21.2 & 1.59 & $1.05-2.40$ & 0.02 & & & \\
\hline \multicolumn{9}{|l|}{ Use of bed net } \\
\hline No & 315 & 21.0 & 1 & & & & & \\
\hline Yes & 431 & 13.7 & 0.60 & $0.40-0.90$ & 0.009 & & & \\
\hline \multicolumn{9}{|c|}{ Intake of AL, preceding 2 weeks } \\
\hline No & 725 & 15.4 & 1 & & & 1 & & \\
\hline Yes & 24 & 54.4 & 6.47 & $2.64-15.9$ & $<0.0001$ & 6.93 & $2.70-17.78$ & $<0.0001$ \\
\hline \multicolumn{9}{|l|}{ Chloroquine in plasma } \\
\hline No & 719 & 14.5 & 1 & & & 1 & & \\
\hline Yes & 28 & 67.9 & 12.48 & $5.19-32.1$ & $<0.0001$ & 17.18 & $6.84-43.16$ & $<0.0001$ \\
\hline
\end{tabular}

n.a., not applicable; MUAC, mid upper arm circumference; RwF, Rwandan Francs; AL, artemether-lumefantrine; OR, odds ratio; aOR, adjusted OR derived from logistic regression including all parameters listed here and following stepwise backward removal of factors not associated in multivariate analysis.

$\mathrm{SE}=0.25 ; P=0.001)$, the main analysis was performed for community children (Table 5 ). In these, anaemia $(\mathrm{Hb}<11 \mathrm{~g} / \mathrm{dL})$ was observed in $34 \%$ and fever in $3 \%$. Parasitaemia was associated with a reduction in mean $\mathrm{Hb}$ of- $2.2 \mathrm{~g} / \mathrm{dL}$, and, age-adjusted, eighteen-fold and five-fold increased odds of anaemia and fever, respectively. These effects were pronounced at increasing parasite density. However, even in submicroscopic infections (all afebrile), mean $\mathrm{Hb}$ was significantly reduced by-1.4 g/dL (Table 5). 
Table 5 Manifestation of malaria in rural Huye subdistrict

\begin{tabular}{|c|c|c|c|c|c|c|c|c|c|}
\hline & \multirow[b]{2}{*}{ No. } & \multicolumn{3}{|c|}{ Fever } & \multicolumn{3}{|c|}{ Anaemia $(\mathrm{Hb}<11 \mathrm{~g} / \mathrm{dl})$} & \multicolumn{2}{|c|}{$\mathrm{Hb}(\mathrm{g} / \mathrm{dL})$} \\
\hline & & $\%$ & $P$ & aOR $(95 \% \mathrm{Cl})$ & $\%$ & $P$ & aOR $(95 \% \mathrm{Cl})$ & Mean & $P$ \\
\hline \multicolumn{10}{|l|}{ Parasitaemia } \\
\hline Absent & 484 & $2.3(11 / 482)$ & & & 28.1 & & & 11.5 & \\
\hline Present & 61 & 11.5 & 0.002 & $\begin{array}{l}4.8 \\
(1.8-13.2)\end{array}$ & 82.0 & $<0.001$ & $\begin{array}{l}17.5 \\
(8.4-36.4)\end{array}$ & 9.3 & $<0.0001$ \\
\hline \multicolumn{10}{|l|}{ Parasite density } \\
\hline None & 484 & $2.3(11 / 482)$ & & & 28.1 & & & 11.5 & \\
\hline$<1000$ & 26 & 7.7 & 0.1 & $\begin{array}{l}3.4 \\
(0.7-16.5)\end{array}$ & 65.4 & 0.0002 & $\begin{array}{l}6.0 \\
(2.5-14.4)\end{array}$ & 9.9 & $<0.0001$ \\
\hline $1000<10000$ & 25 & 12.0 & 0.01 & $\begin{array}{l}4.8 \\
(1.2-18.9)\end{array}$ & 96.0 & $<0.0001$ & $\begin{array}{l}105.7 \\
(13.8-812.5)\end{array}$ & 9.1 & $<0.0001$ \\
\hline$>=10000$ & 10 & 20.0 & 0.005 & $\begin{array}{l}8.6 \\
(1.6-46.9)\end{array}$ & 90.0 & 0.003 & $\begin{array}{l}40.5 \\
(4.9-336.7)\end{array}$ & 8.3 & $<0.0001$ \\
\hline \multicolumn{10}{|c|}{ P. falciparum infection, PCR } \\
\hline Absent & 457 & $2.4(11 / 455)$ & & & 26.7 & & & 11.6 & \\
\hline Present & 88 & 8.0 & 0.008 & $\begin{array}{l}3.0 \\
(1.1-8.1)\end{array}$ & 72.7 & $<0.0001$ & $\begin{array}{l}10.8 \\
(6.1-18.9)\end{array}$ & 9.6 & $<0.0001$ \\
\hline \multicolumn{10}{|l|}{ Staged infection } \\
\hline None & 453 & $2.4(11 / 451)$ & & & 26.5 & & & 11.6 & \\
\hline Submicroscopic & 31 & 0 & 1 & $\begin{array}{l}0 \\
(0-\infty)\end{array}$ & 51.6 & 0.004 & $\begin{array}{l}4.0 \\
(1.9-8.8)\end{array}$ & 10.2 & 0.0009 \\
\hline Microscopic & 61 & 11.5 & 0.004 & $\begin{array}{l}4.4 \\
(1.6-12.2)\end{array}$ & 82.0 & $<0.0001$ & $\begin{array}{l}20.3 \\
(9.6-42.6)\end{array}$ & 9.3 & $<0.0001$ \\
\hline
\end{tabular}

aOR, age-adjusted odds ratio. $95 \% \mathrm{Cl}, 95 \%$ confidence interval. $\mathrm{Hb}$, haemoglobin concentration. Hb levels are compared by the non-parametric Mann Whitney $\mathrm{U}$ test.

In children attending the health centre, there were trends only of an association between anaemia and $P$. falciparum infection by PCR (age-adjusted OR (95\% CI), 2.77 (0.90-8.55), $P=0.08$ ) or microscopically visible parasitaemia (2.69 (0.80-9.0), $P=0.11)$. In contrast, at the district hospital, these figures yielded statistical significance (PCR, 4.62 (1.34-15.95), $P=0.02$; microscopy, 10.3 (1.89-55.88), $P=0.007)$. For fever, the opposite was seen: Parasitaemia, e.g. detected by microscopy, increased the risk of fever nine times in the health centre (age-adjusted OR (95\% CI), 8.88 (2.54-31.04), $P=$ $0.0006)$ but showed no association in the hospital (1.27 (0.30-5.39), $P=0.74)$.

\section{Discussion}

Malaria transmission in Rwanda varies widely. Traditionally, the central plateau (altitude 1,500 to $1,800 \mathrm{~m}$ ) is considered as one of four distinct ecological zones with overall P. falciparum prevalence rates of 5\% to 15\% [17]. While transmission in Rwanda is regarded to be stable with seasonal peaks in the valleys and unstable (and potentially epidemic-prone) at higher altitude [18], a linear correlation between altitude and transmission would be oversimplified: in a recent tabulation of the years 2001-2007, endemicity at 1,600-2,000 $\mathrm{m}$ above sea level ranged from hypo-to holoendemic and annual malaria incidences (presumed and confirmed) from 2.4 to 20.4 per 1000 capita
[17]. Likely, these figures have declined in recent years $[4,7,8]$. Data from the 2007-2008 Demographic and Health Survey (DHS) [19] indicate that in $>4,600$ children $<5$ years of age and sampled across Rwanda, $2.6 \%$ had malaria infection based on rapid Plasmodium lactate dehydrogenase tests. In the present study from the vicinity of Butare (altitude, $1,768 \mathrm{~m}$ ), 11\% and 16\% of community children were infected with $P$. falciparum based on microscopy and PCR, respectively. Data from the 2010 Rwanda Health Management Information System indicate that 13.4\% of patients attending health facilities in the Huye district had microscopically confirmed malaria. In the present study, this figure was $12.3 \%$.

Given the scarcity of published community-based data, the reasons for the discrepancy in the prevalence of infection reported in the DHS and observed in the present study are difficult to appraise. Higher sensitivity of PCR as compared to microscopy or rapid test devices $[20,21]$ may partially be involved. Selection bias during recruitment at home, e.g., due to preferential presentation by the parents of children with (a recent history of) sickness cannot completely be excluded. However, recruitment teams were instructed to select children from households randomly and into pre-defined age strata. Also, most infections in the communities were asymptomatic. One limitation of the present study is its cross-sectional nature by which e.g., seasonal fluctuations are not 
reproduced. Geographical variation of infection between villages was evident (Figure 2) but attempts to relate this to e.g. altitude or proximity to a water stream, failed. Likely involved, most villages comprise homesteads scattered in the hills rather than agglomerated settlements [22]. Other parameters in the present study, e.g. selfreported bed net use, socio-economic factors, anaemia, were largely in the reported range $[4,19,22]$. Thus, the present data are not representative for the central plateau, let alone Rwanda, but rather provide a detailed and up-to-date picture of $P$. falciparum infection in southern highland communities and in health facilities serving this population. In contrast, routine health facility based surveillance has clear limitations in providing complete or representative data on e.g., malaria in the community, also because patients lacking access or choosing alternatives are not registered [23]. A low health insurance coverage $(43 \%)$ in the communities may have deterred parents from seeking formal health care. Such, in turn, could have lead to an over-estimation of disease burden at the community level as compared to the end of the year when more have paid their fees. Nevertheless, only $20 \%$ of African children with suspicion of malaria are considered to come to the attention of any formal health system [24], a figure that might have improved in recent years [4]. Community-based surveys, despite their local limitations, thus provide essential information, also for control campaign monitoring $[10,11]$.

In the communities, infection prevalence increased from $10 \%$ to almost $25 \%$ at four years of age, which was not accompanied by a decline in parasite density with age or increase in MOI. The additional age-dependent increase in malaria and trend for declining asymptomatic infections indicate that semi-immunity did not develop to the extent observed in highly endemic areas [25-27]. In line with this, $P$. falciparum infection including submicroscopic ones had an impact on Hb levels, which exceeds the one commonly seen in children in highendemicity areas [27-29]. On the other hand, only a quarter of parasitaemic children had malaria, suggesting a majority of asymptomatic infections. Irrespective of the erratic nature of fever in the definition of malaria, the presence of (usually undetected) asymptomatic P. falciparum infections has important implications for malaria control in highland areas. In two sites in highland Kenya, both, high and low levels of asymptomatic P. falciparum infections have been observed among children and adults $[30,31]$. Studies from Ghana and Sudan indicate that asymptomatic infections can persist for a year or longer [32,33]; in highland Kenya the median duration in children aged 5-9 years was five months [31]. Gametocyte carriage was not consistently assessed in the present study but appeared to be low. Nevertheless, individuals with low level, long-lasting, and asymptomatic infections form a major reservoir for transmission [21,34,35]. In situations of increased rainfall, higher temperatures, or changed land use such asymptomatic infections may give rise to epidemics which have increased in frequency and intensity in East Africa during the last two decades [36-39]. Targeted antimalarial treatment even of asymptomatic children may thus be a justifiable part of malaria control in highland areas. However, the differing findings on the level of asymptomatic parasite carriage in the present and the two Kenyan studies [30,31] illustrate that results may not be readily extrapolated.

At the health facilities, roughly half of the children had respiratory tract infections or gastrointestinal problems. Every sixth child at the health centre had malaria and every fifth was $P$. falciparum infected. Irrespective of the better socio-economic status of the patients' families as compared to the communities (Table 2) this indicates that malaria is among the top three reasons to seek primary health care in this area. At eight percent prevalence, malaria was of lesser importance at the district hospital which receives referrals from several health centres and patients bypassing the referral system by self-paying. There, $38 \%$ of the patients were reportedly pre-treated including $6 \%$ with AL. The validity of (malaria) treatment histories frequently is questionable [40], and no data on the dose and duration of treatment were collected in the present study. Nevertheless, the finding that recent AL treatment was positively associated with current malaria is remarkable. This is suggestive of recurrence of parasitaemia following treatment. In fact, drug resistance markers associated with reappearing parasitaemia following AL treatment tended to be increased in these infections (Zeile et al , unpublished observations). Latest cure rates of AL in Rwanda from 2006 have been reported as 97\% [41]. Nevertheless, against the background of intense AL drug pressure in Rwanda in recent years, this finding underlines the necessity of the upcoming re-evaluation of the drug's efficacy in this country.

Intake of chloroquine was stated by none of the respondents but the drug was present in plasma in $1.4 \%$ and $15.4 \%$ of non-infected and infected children, respectively. With the assay applied, chloroquine intake can be detected for several weeks, depending on the dose; cross-reactivity with amodiaquine is negligible [16]. Likely, the finding of an increased infection prevalence in chloroquine positive children reflects the combination of previous home-treatment and persisting or recrudescent parasites due to intense chloroquine resistance which is prevalent in Rwanda [42].

Among the age-adjusted risk factors for $P$. falciparum infection was a decreasing MUAC. This crude proxy parameter for malnutrition was, however, not associated with malaria itself. Chronic malnutrition affects every second child in Rwanda [19] and compromises anti- 
pathogen immunity [43]. Lacking effect on malaria morbidity as observed in the present study corresponds with previous findings $[29,44]$ but contrasts with others $[28,45,46]$. Possibly, the differential effect on infection and malaria depends on the specific yet unknown type of malnutrition in the study area. Considering the modifiable nature of this risk factor and Rwanda's recently renewed commitment to fight malnutrition, more research into this field is needed.

At variable statistical significance, several parameters reflecting low socio-economic status were associated with increased risks of infection. Remarkably, possession of a radio or a bicycle were independently associated predictors of reduced $P$. falciparum prevalence. This may reflect increased access to malaria-related information, improved awareness and increased usage of curative services. Given the latter is true, this points to accomplishable ways of reducing $P$. falciparum infection in the area, i.e. health communication and education.

Lastly, although self-reported bed net usage was in the previously observed range $[4,19,22]$, the detectable impact was modest and non-significant in multivariate analysis. This finding points to deficits in an established mean of malaria prevention the efficacy of which has been confirmed in many studies $[4,7,10,11]$. The reasons may be diverse and rather involve caregivers' beliefs about causation and vulnerability as well as obstacles in translating knowledge into behaviour than insecticide resistance [47-49]. Nevertheless, these actual reasons need to be assessed at the community-level and subsequent campaigns should address potential obstacles to promote consistent and correct use.

\section{Conclusions}

In this community and facility based survey on malariologic parameters in southern highland Rwanda, P. falciparum infection was observed in one out of six children under five years of age, without much variation between community and health facilities. While facility-based, most infections were symptomatic, the opposite was seen in the communities. These seemingly asymptomatic infections greatly contributed to anaemia and form an unrecognized source of transmission in the epidemicprone highland area. Improved nutrition, identification and elimination of causes of low bed net effectiveness, and reinforced health education are promising and tangible measures to further reduce $P$. falciparum in this area of Rwanda. In parallel, community-based surveillance of malaria should be included to monitor the progress of malaria control.

\section{Acknowledgements}

The authors thank the children's families for participation in this study, and the staff at Sovu health centre, Kabutare district hospital, and CHUB central laboratory for assistance and cooperation. The active assistance by the Rwanda office of the German Development Cooperation (GIZ) is gratefully acknowledged. This study was supported by the German Federal Ministry for Economic Cooperation and Development via the ESTHER programme (Ensemble pour une Solidarité Thérapeutique Hospitalière en Réseau ). This work forms part of the theses of CSt and IZ.

\section{Author details}

${ }^{1}$ Butare University Teaching Hospital, Faculty of Medicine, National University of Rwanda, Butare, Rwanda. ${ }^{2}$ Institute of Tropical Medicine and International Health, Charité-University Medicine Berlin, Berlin, Germany. ${ }^{3}$ German Development Cooperation (GIZ), Health Programme, Kigali, Rwanda. ${ }^{4}$ Division of Infectious Diseases, Tropical Medicine and AIDS, Academic Medical Centre, Amsterdam, The Netherlands. ${ }^{5}$ National Malaria Control Programme/Malaria Unit, TRAC Plus, Kigali, Rwanda.

\section{Authors' contributions}

JBG, FPM, AM, and GH designed the study. JBG, CSt, CSh, NCA, CHL, and CK were responsible for patient recruitment, clinical and laboratory examinations, and logistics. IZ did the PCR analyses, TEA the ELISA assays, and ID, JBG, AU and FPM the statistical analyses. JBG and FPM wrote the paper with major contributions of the other authors. All authors read and approved the final manuscript.

\section{Competing interests}

The authors declare that they have no competing interests.

Received: 2 March 2011 Accepted: 18 May 2011 Published: 18 May 2011

\section{References}

1. Guerra CA, Gikandi PW, Tatem AJ, Noor AM, Smith DL, Hay SI, Snow RW: The limits and intensity of Plasmodium falciparum transmission: implications for malaria control and elimination worldwide. PLOS Med 2008, 5:e38.

2. O'Meara WP, Bejon P, Mwangi TW, Okiro EA, Peshu N, Snow RW, Newton CRJC, Marsh K: Effect of a fall in malaria transmission on morbidity and mortality in Kilifi, Kenya. Lancet 2008, 372:1555-1562.

3. Roll Back Malaria: The global malaria action plan. For a malaria-free world Geneva, Switzerland; 2008.

4. World Health Organization: World malaria report 2009 [http://www.who.int/ malaria/world_malaria_report_2009/en/index.html].

5. D'Acremont $V$, Lengeler C, Genton B: Reduction in the proportion of fevers associated with Plasmodium falciparum parasitaemia in Africa: a systematic review. Malar J 2010, 9:240.

6. Griffin JT, Hollingsworth TD, Okell LC, Churcher TS, White M, Hinsley W, Bousema T, Drakeley CJ, Ferguson NM, Basáñez MG, Ghani AC: Reducing Plasmodium falciparum malaria transmission in Africa: a model-based evaluation of intervention strategies. PLoS Med 2010, 7:e1000324.

7. Otten M, Aregawi M, Were W, Karema C, Medin A, Bekele W, Jima D, Gausi K, Komatsu R, Korenromp E, Low-Beer D, Grabowsky M: Initial evidence of reduction of malaria cases and deaths in Rwanda and Ethiopia due to rapid scale-up of malaria prevention and treatment. Malar J 2009, 8:14

8. Sievers AC, Lewey J, Musafiri P, Franke MF, Bucyibaruta BJ, Stulac SN, Rich ML, Karema C, Daily JP: Reduced paediatric hospitalizations for malaria and febrile illness patterns following implementation of community-based malaria control programme in rural Rwanda. Malar $J$ 2008, 7:167.

9. Tiono AB, Kaboré Y, Traoré A, Convelbo N, Pagnoni F, Sirima SB: Implementation of home based management of malaria in children reduces the work load for peripheral health facilities in a rural district of Burkina Faso. Malar J 2008, 7:201

10. Rowe AK, Kachur SP, Yoon SS, Lynch M, Slutsker L, Steketee RW: Caution is required when using health facility-based data to evaluate the health impact of malaria control efforts in Africa. Malar J 2009, 8:209.

11. Rowe AK, Steketee RW, Arnold F, Wardlaw T, Basu S, Bakyaita N, Lama M, Winston CA, Lynch M, Cibulskis RE, Shibuya K, Ratcliffe AA, Nahlen BL: Roll Back Malaria Monitoring and Evaluation Reference Group: Viewpoint: evaluating the impact of malaria control efforts on mortality in subSaharan Africa. Trop Med Int Health 2007, 12:1524-1539. 
12. Twahirwa A: Sharing the burden of sickness: mutual health insurance in Rwanda. Bull World Health Organ 2008, 86:823-824.

13. Ministry of Health, Kigali, Rwanda: Protocoles des Services de Santé [http://www.moh.gov.rw/index.php?option=com_docman\&task=cat_ view\&gid=62\&ltemid=14]

14. Rubio JM, Post RJ, van Leeuwen WM, Henry MC, Lindergard G, Hommel M: Alternative polymerase chain reaction method to identify Plasmodium species in human blood samples: the semi-nested multiplex malaria PCR (SnM-PCR). Trans R Soc Trop Med Hyg 2002, 96(Suppl 1):S199-204.

15. Snounou G, Zhu X, Siripoon N, Jarra W, Thaithong S, Brown KN, Viriyakosol S: Biased distribution of msp1 and msp2 allelic variants in Plasmodium falciparum populations in Thailand. Trans $R$ Soc Trop Med Hyg 1999, 93:369-374.

16. Eggelte TA: Production of monoclonal antibodies against antimalarial drugs for use in immunoassays. In The validation of chemical and immunochemical tests for antimalarials in body fluids. Edited by: Navaratnam V, Payne D. International Monograph Series 3. Penang, Malaysia: Universiti Sains Malaysia; 1990:35-63.

17. Rulisa S, Mens PF, Karema C, Schallig HD, Kaligirwa N, Vyankandondera J, de Vries PJ: Malaria has no effect on birth weight in Rwanda. Malar J 2009, 8:194

18. Rwagacondo CE, Niyitegeka F, Sarushi J, Karema C, Mugisha V, Dujardin JC, Van Overmeir C, van den Ende J, D'Alessandro U: Efficacy of amodiaquine alone and combined with sulfadoxine-pyrimethamine and of sulfadoxine pyrimethamine combined with artesunate. Am J Trop Med Hyg 2003, 68:743-747.

19. Ministry of Health of Rwanda: Rwanda Interim Demographic and Health Survey 2007-2008 Ministry of Health of Rwanda, Kigali, Rwanda; 2009 [http:// www.measuredhs.com/pubs/pdf/FR215/FR215.pdf].

20. Rakotonirina $H$, Barnadas C, Raherijafy R, Andrianantenaina $H$, Ratsimbasoa A, Randrianasolo L, Jahevitra M, Andriantsoanirina V, Ménard D: Accuracy and reliability of malaria diagnostic techniques for guiding febrile outpatient treatment in malaria-endemic countries. Am J Trop Med Hyg 2008, 78:217-221

21. Okell LC, Ghani AC, Lyons E, Drakeley CJ: Submicroscopic infection in Plasmodium falciparum -endemic populations: a systematic review and meta-analysis. J Infect Dis 2009, 200:1509-1517.

22. National Institute of Statistics of Rwanda: Districts Baseline Survey: Huye. 2007 National Institute of Statistics of Rwanda, Kigali, Rwanda; 2009 [http:// statistics.gov.rw/index.php?

option=com_content\&task=view\&id=158\&ltemid=321].

23. Oum S, Chandramohan D, Cairncross S: Community-based surveillance: a pilot study from rural Cambodia. Trop Med Int Health 2005, 10:689-697.

24. Breman JG: The ears of the hippopotamus: manifestations, determinants, and estimates of the malaria burden. Am J Trop Med Hyg 2001, 64(1-2 Suppl):1-11.

25. Trape JF, Rogier C, Konate L, Diagne N, Bouganali H, Canque B, Legros F, Badji A, Ndiaye G, Ndiaye P, Brahimi K, Faye O, Pereira Da Silva L: The Dielmo project: a longitudinal study of natural malaria infection and the mechanisms of protective immunity in a community living in a holoendemic area of Senegal. Am J Trop Med Hyg 1994, 51:123-137.

26. Smith T, Felger I, Tanner M, Beck HP: Premunition in Plasmodium falciparum infection: insights from the epidemiology of multiple infections. Trans R Soc Trop Med Hyg 1999, 93(Suppl 1):59-64.

27. Mmbando BP, Segeja MD, Msangeni HA, Sembuche SH, Ishengoma DS, Seth MD, Francis F, Rutta AS, Kamugisha ML, Lemnge MM: Epidemiology of malaria in an area prepared for clinical trials in Korogwe, northeastern Tanzania. Malar J 2009, 8:165

28. Ehrhardt S, Burchard GD, Mantel C, Cramer JP, Kaiser S, Kubo M, Otchwemah RN, Bienzle U, Mockenhaupt FP: Malaria, anemia, and malnutrition in african children-defining intervention priorities. J Infect Dis 2006, 194:108-114.

29. Danquah I, Dietz E, Zanger $P$, Reither K, Ziniel P, Bienzle U, Mockenhaupt FP: Reduced efficacy of intermittent preventive treatment of malaria in malnourished children. Antimicrob Agents Chemother 2009, 53:1753-1759.

30. John CC, McHugh MM, Moormann AM, Sumba PO, Ofulla AV: Low prevalence of Plasmodium falciparum infection among asymptomatic individuals in a highland area of Kenya. Trans R Soc Trop Med Hyg 2005, 99:780-786.
31. Baliraine FN, Afrane YA, Amenya DA, Bonizzoni M, Menge DM, Zhou G, Zhong D, Vardo-Zalik AM, Githeko AK, Yan G: High prevalence of asymptomatic Plasmodium falciparum infections in a highland area of western Kenya: a cohort study. J Infect Dis 2009, 200:66-74.

32. Franks S, Koram KA, Wagner GE, Tetteh K, McGuinness D, Wheeler JG, Nkrumah F, Ranford-Cartwright L, Riley EM: Frequent and persistent, asymptomatic Plasmodium falciparum infections in African infants, characterized by multilocus genotyping. J Infect Dis 2001, 183:796-804

33. Nassir E, Abdel-Muhsin AM, Suliaman S, Kenyon F, Kheir A, Geha $H_{\text {, }}$ Ferguson HM, Walliker D, Babiker HA: Impact of genetic complexity on longevity and gametocytogenesis of Plasmodium falciparum during the dry and transmission-free season of eastern Sudan. Int J Parasitol 2005, 35:49-55.

34. Bousema JT, Gouagna LC, Drakeley CJ, Meutstege AM, Okech BA, Akim IN Beier JC, Githure Jl, Sauerwein RW: Plasmodium falciparum gametocyte carriage in asymptomatic children in western Kenya. Malar J 2004, 3:18.

35. Alves FP, Gil LH, Marrelli MT, Ribolla PE, Camargo EP, Da Silva LH: Asymptomatic carriers of Plasmodium spp. as infection source for malaria vector mosquitoes in the Brazilian Amazon. J Med Entomol 2005, 42:777-779.

36. Lindblade KA, Walker ED, Onapa AW, Katungu J, Wilson ML: Land use change alters malaria transmission parameters by modifying temperature in a highland area of Uganda. Trop Med Int Health 2000, 5:263-74.

37. Hay SI, Were EC, Renshaw M, Noor AM, Ochola SA, Olusanmi I, Alipui N, Snow RW: Forecasting, warning, and detection of malaria epidemics: a case study. Lancet 2003, 361:1705-1706.

38. Shanks GD, Hay SI, Omumbo JA, Snow RW: Malaria in Kenya's western highlands. Emerg Infect Dis 2005, 11:1425-1432.

39. Checchi F, Cox J, Balkan S, Tamrat A, Priotto G, Alberti KP, Guthmann JP: Malaria epidemics and interventions, Kenya, Burundi, southern Sudan, and Ethiopia, 1999-2004. Emerg Infect Dis 2006, 12:1477-1485.

40. Nwanyanwu OC, Redd SC, Ziba C, Luby SP, Mount DL, Franco C, Nyasulu Y, Chitsulo L: Validity of mother's history regarding antimalarial drug use in Malawian children under five years old. Trans R Soc Trop Med Hyg 1996, 90:66-8.

41. Fanello Cl, Karema C, van Doren W, Van Overmeir C, Ngamije D, D'Alessandro U: A randomised trial to assess the safety and efficacy of artemether-lumefantrine (Coartem) for the treatment of uncomplicated Plasmodium falciparum malaria in Rwanda. Trans $R$ Soc Trop Med Hyg 2007, 101:344-350

42. East African Network for Monitoring Antimalarial Treatment (EANMAT): The efficacy of antimalarial monotherapies, sulphadoxine-pyrimethamine and amodiaquine in East Africa: implications for sub-regional policy. Trop Med Int Health 2003, 8:860-867.

43. Schaible UE, Kaufmann SH: Malnutrition and infection: complex mechanisms and global impacts. PLoS Med 2007, 4:e115.

44. Müller O, Garenne M, Kouyaté B, Becher H: The association between protein-energy malnutrition, malaria morbidity and all-cause mortality in West African children. Trop Med Int Health 2003, 8:507-511.

45. Deen $J$, Walraven GE, von Seidlein L: Increased risk for malaria in chronically malnourished children under 5 years of age in rural Gambia. J Trop Pediatr 2002, 48:78-83.

46. Caulfield LE, Richard SA, Black RE: Undernutrition as an underlying cause of malaria morbidity and mortality in children less than five years old. Am J Trop Med Hyg 2004, 71(2 Suppl):55-63.

47. Aikins MK, Pickering $H$, Greenwood BM: Attitudes to malaria, traditional practices and bednets (mosquito nets) as vector control measures: a comparative study in five west African countries. J Trop Med Hyg 1994, 97:81-86.

48. Okrah J, Traoré C, Palé A, Sommerfeld J, Müller O: Community factors associated with malaria prevention by mosquito nets: an exploratory study in rural Burkina Faso. Trop Med Int Health 2002, 7:240-248.

49. Curtis CF, Maxwell CA, Magesa SM, Rwegoshora RT, Wilkes TJ: Insecticidetreated bed-nets for malaria mosquito control. J Am Mosa Control Assoc 2006, 22:501-506.

doi:10.1186/1475-2875-10-134

Cite this article as: Gahutu et al:: Prevalence and risk factors of malaria among children in southern highland Rwanda. Malaria Journal 2011 10:134. 